\title{
The control of viral infection by tripartite motif proteins and cyclophilin A Greg J Towers*
}

Address: MRC Centre for Medical Molecular Virology, Department of Infection, Royal Free and University College London Medical School, 46 Cleveland Street, London, W1T4JF, UK

Email: Greg J Towers* - g.towers@ucl.ac.uk

* Corresponding author

Published: 12 June 2007

Retrovirology 2007, 4:40 doi:10.1 I86/1742-4690-4-40

This article is available from: http://www.retrovirology.com/content/4/I/40

This is an Open Access article distributed under the terms of the Creative Commons Attribution License (http://creativecommons.org/licenses/by/2.0), which permits unrestricted use, distribution, and reproduction in any medium, provided the original work is properly cited.

\begin{abstract}
The control of retroviral infection by antiviral factors referred to as restriction factors has become an exciting area in infectious disease research. TRIM5 $\alpha$ has emerged as an important restriction factor impacting on retroviral replication including HIV-I replication in primates. TRIM5 $\alpha$ has a tripartite motif comprising RING, B-Box and coiled coil domains. The antiviral $\alpha$ splice variant additionally encodes a B30.2 domain which is recruited to incoming viral cores and determines antiviral specificity. TRIM5 is ubiquitinylated and rapidly turned over by the proteasome in a RING dependent way. Protecting restricted virus from degradation, by inhibiting the proteasome, rescues DNA synthesis, but not infectivity, indicating that restriction of infectivity by TRIM $5 \alpha$ does not depend on the proteasome but the early block to DNA synthesis is likely to be mediated by rapid degradation of the restricted cores. The peptidyl prolyl isomerase enzyme cyclophilin $A$ isomerises a peptide bond on the surface of the HIV-I capsid and impacts on sensitivity to restriction by TRIM5 $\alpha$ from Old World monkeys. This suggests that TRIM $5 \alpha$ from Old World monkeys might have a preference for a particular capsid isomer and suggests a role for cyclophilin $A$ in innate immunity in general. Whether there are more human antiviral TRIMs remains uncertain although the evidence for TRIMI9's (PML) antiviral properties continues to grow. A TRIM5-like molecule with broad antiviral activity in cattle suggests that TRIM mediated innate immunity might be common in mammals. Certainly the continued study of restriction of viral infectivity by antiviral host factors will remain of interest to a broad audience and impact on a variety of areas including development of animal models for infection, development of viral vectors for gene therapy and the search for novel antiviral drug targets.
\end{abstract}

\section{Background}

The control of viral infection by intracellular antiviral proteins referred to as restriction factors has become an important and challenging focus of infectious disease research. A clearer understanding of the role of restriction factors in immunity and the control of retroviral replication promises to reveal details of host virus relationships, allow improvement of animal models of infection, iden- tify targets for antiviral therapies, and further facilitate the use of viral vectors for clinical and investigative gene delivery. The tripartite motif protein TRIM $5 \alpha$ has recently emerged as an important restriction factor in mammals blocking infection by retroviruses in a species-specific way. Early evidence for TRIM5 $\alpha$ 's antiviral activity included the species-specific infectivity of retroviral vectors, even when specific envelope/receptor requirements 
were obviated by the use of the VSV-G envelope. Notable examples include the poor infectivity of certain murine leukemia viruses (MLV) on cells from humans and primates and the poor infectivity of HIV-1 on cells from Old World monkeys [1-3]. The notion that a dominant antiviral factor was responsible was suggested by the demonstration that the block to infection could be saturated, or abrogated, by high doses of retroviral cores [4-6]. The putative human antiviral factor was named Ref 1 and the simian factor Lv1 $[1,6]$. TRIM5 $\alpha$ was identified in 2004 by screening rhesus cDNAs for those with antiviral activity against HIV-1 [7]. Shortly after, several groups demonstrated that Ref1 and Lv1 were encoded by species-specific variants of TRIM $5 \alpha$ [8-11]. TRIM5 $\alpha$ therefore represents a hitherto undescribed arm of the innate immune system, blocking infection by an incompletely characterised mechanism. Its expression is induced by interferon via an IRF3 site in the TRIM5 promoter linking it to the classical innate immune system [12].

\section{The tripartite motif}

TRIM5 has a tripartite motif, also known as an RBCC domain, comprising a RING domain, a B Box 2 domain and a coiled coil $[13,14]$. The RING is a zinc-binding domain, typically involved in specific protein-protein interactions. Many RING domains have E3 ubiquitin ligase activity and TRIM5 can mediate RING dependent auto-ubiquitinylation in vitro [15]. B boxes are of 2 types, either B-box1 or B-box2 and TRIM5 encodes a B-box2. Bboxes have a zinc-binding motif and are putatively involved in protein-protein interactions. The two types of B-box have distinct primary sequence but similar tertiary structures and are structurally similar to the RING domain. This suggests that they may have evolved from a common ancestral fold, and perhaps have a similar function, such as ubiquitin ligation $[16,17]$. It is also possible that B-Boxes contributes to ligation specificity, ie have E4 activity $[16,17]$. The coiled-coil is involved in homo- and hetero-multimerisation of TRIM proteins $[14,18]$. TRIM5 exists as a trimer with the coiled coil facilitating homo and hetero multimerisation with related TRIM proteins [1820].

TRIM5 RNA is multiply spliced, generating a family of isoforms, each shorter from the $\mathrm{C}$ terminus. The longest, TRIM $5 \alpha$, encodes a C terminal B30.2 domain that interacts directly with viral capsid and determines antiviral specificity $[18,21,22]$. The shorter isoforms, TRIM $5 \gamma$ and TRIM $5 \delta$, do not have B30.2 domains and act as dominant negatives to TRIM $5 \alpha$ and rescue restricted infectivity when over-expressed $[7,23]$. It is assumed that the shorter forms form heteromultimers via the coiled coil and titrate the viral binding B30.2 domains. It is therefore possible that TRIM5's antiviral activity is regulated by splicing.
The B30.2 domain comprises a combination of a PRY motif followed by a SPRY motif [24]. Whilst SPRY domains are evolutionary ancient, B30.2 domains, found in butyrophilin and TRIM proteins, appeared more recently. There is unlikely to be a precise function for B30.2 domains, rather they are involved in protein-protein interactions such as substrate recognition. A series of TRIM5 mutagenesis studies demonstrated that the TRIM5 B30.2 domain determines antiviral specificity and defined the specific regions of the $\mathrm{B} 30.2$ responsible $[18,21,22,25,26]$. In vitro capsid/TRIM5 binding assays have been developed and these demonstrate that, at least in the case of wild type TRIM $5 \alpha$ proteins, binding correlates well with the ability to restrict infection $[27,28]$.

The recent solution of the structure of several B30.2 domains allows us to interpret the conservation and variation between TRIM5 B30.2 domains [29-31]. The structures indicate that the $\mathrm{B} 30.2$ core is formed from a distorted 2-layer beta sandwich with the beta strands in an anti-parallel arrangement. Extending from the core are a series of loops and it is these surface loop structures that vary between the TRIM 5 sequences from each primate and between different B30.2 domains of TRIM5 homologues. The loops form 3 or 4 variable regions, all of which appear to impact on antiviral specificity [32]. The TRIM21 structure in complex with its ligand, IgG Fc indicates that there are 2 binding surfaces, one in the PRY (V1) and 1 in the SPRY (V2-V3) and this is likely to be true for TRIM5 $\alpha$.

\section{TRIM5 and the Red Queen}

B30.2 mutagenesis studies, as well as sequence analysis of TRIM5 $\alpha$ from related primates, suggested that the differences defining anti-viral specificity are concentrated in patches in the B30.2 domain [33]. The patches, which correspond to the surface loops, have been under very strong positive selection as evidenced by a high $\mathrm{dN}$ :dS ratio. $\mathrm{dN}$ : dS ratios have been calculated by comparing TRIM5 sequences from primates and comparing the number of differences that lead to a change in the protein sequence (non synonomous, $\mathrm{dN}$ ) to the number of differences that do not (synonomous, dS). A high ratio indicates positive selection and is evidence of the host-pathogen arms race known as the Red Queen hypothesis [34]. This phenomenon, named after Lewis Carroll's Red Queen who claimed 'It takes all the running you can do to keep in the same place', refers to the selection driven genetic change that occurs in both host and pathogen as each alternately gains the advantage. Whether selection pressure on TRIM5 has been from pathogenic retroviruses or from endogenous retroviruses and retrotransposons is unclear. The relative youth of lentiviruses, as compared to other retroviruses and endogenous elements, is thought to preclude them from impacting on TRIM5 selection, although the discovery of an endogenous lentivirus in rabbits [35] has 
recently extended their age from less than 1 million years to greater than 7 million years and it certainly seems possible that this age will extend further as we better understand lentiviral history.

The other side of the Red Queen's arms race is the change in the retroviral capsids to escape restriction by TRIM5. TRIM5 molecules can generally restrict widely divergent retroviruses including gamma retroviruses as well as lentiviruses. For example Agm and bovine TRIMs restrict MLVN, HIV-1, HIV-2 and SIVmac [36-38]. It is now clear that retroviral capsid structures are conserved and capsid hexamers are found in both lentiviruses and gamma retroviruses $[39,40]$ so we imagine that the TRIMs recognise a conserved shape. Paradoxically, point mutants can often escape strong restriction. MLV-N CA R110E escapes human, simian and bovine TRIMs, SIVmac CA QQ8990LPA escapes rhesus and squirrel monkey TRIM5s and HIV-1 G89V escapes owl monkey TRIMCyp [1,38,41-44]. It therefore remains unclear how TRIM5 can be effective if a small number of changes in CA can rescue infectivity, especially given that retroviral capsid sequences appear quite plastic.

\section{The antiviral mechanism}

We are beginning to understand TRIM5 $\alpha$ 's antiviral mechanism. TRIM $5 \alpha$ is trimeric $[19,45]$ and interacts with hexameric capsids [46]. TRIM5 $\alpha$ is ubiquitinylated within cells and is rapidly turned over by the proteasome in a RING domain dependent way suggesting that autoubiquitinylation might drive this process $[15,47]$. We imagine that the rapid turnover of TRIM $5 \alpha$ and presumably TRIM $5 \alpha$-virus complexes leads to an early block to infection, before the virus has had the opportunity to reverse transcribe (Figure 1A). This notion is supported by the observation that inhibition of the proteasome during restricted infection allows the virus to reverse transcribe, when it is protected from degradation $[48,49]$ (Figure 1B). However, infection is not rescued by inhibition of the proteasome, indicating that the TRIM $\alpha$-virus complex remains uninfectious, even when protected from degradation. How exactly TRIM $5 \alpha$ renders the virus uninfectious remains unclear, but it may be that by simply coating the core with multivalent complexes TRIM $5 \alpha$ trimers are able to disrupt the rearrangement/uncoating and or trafficking required to continue to the nucleus and to integrate. Other possibilities include TRIM5 $\alpha$ rapidly uncoating incoming HIV-1 capsids. In fact, this has been observed using an assay of capsid density to measure uncoating $[46,50]$ and it will be interesting perform this assay in the presence and absence of proteasome inhibitors to address whether the proteasome has a role this process. Proteasome independent degradation of capsids by TRIM $5 \alpha$ has also been described [51]. Importantly, DNA circles remain inhibited, even in the absence of proteasome activity, sug- gesting that the restricted TRIM $5 \alpha$-virus complex cannot access the nucleus. [48,49] (Fig 1). It is possible that these observations indicate several independent antiviral activities of TRIM $5 \alpha$ but we prefer the interpretation that there are several possible fates for a restricted virion. It may be degraded by the proteasome, it may inappropriately uncoat, or it may remain intact, make DNA but not have access to the nucleus. The different fates are likely to be influenced by factors such as the particular virus, the particular TRIM $5 \alpha$ as well as virus dose and TRIM5 $\alpha$ expression levels and the cellular background. Understanding the contribution of these activities to restriction by TRIM5 $\alpha$ will require further study but the field continues to make steady progress.

\section{A Role for Cyclophilin A in restriction}

The relationship between Cyclophilin A (CypA) and HIV1 has a long history. CypA is a peptidyl prolyl isomerase that performs cis/trans isomerisation of proline peptide bonds in sensitive proteins. CypA interacts with gag in infected cells leading to its recruitment into nascent HIV1 virions [52,53]. Recent data has shown that CypA also interacts with incoming HIV-1 cores in newly infected cells and that this interaction is more important for infectivity than that occurring as cores assemble [42,54-56]. This may be because only about $10 \%$ of the capsid molecules in the core recruit a CypA molecule into the virion $[52,53]$. CypA performs cis/trans isomerisation at CA G89-P90 on the outer surface of the capsid $[57,58]$ and this leads to changes in infectivity. In Old World monkey (OWM) cells CypA decreases HIV-1 infectivity, but only in the presence of TRIM5 $\alpha$ [59-61]. Blocking CypA activity using the immunosuppressant competitive inhibitor of CypA cyclosporine A (CSA), or reducing CypA expression with small interfering RNA, reduces the susceptibility of HIV-1 to restriction by OWM TRIM5 and rescues HIV-1 infectivity.

In human cells the interaction between incoming HIV-1 cores and CypA is important for maximal infectivity. Preventing this interaction reduces HIV-1 infectivity independently of TRIM5 expression [59,62]. It is suspected that in the absence of CypA activity, HIV-1 gets restricted by a TRIM 5 independent antiviral activity. This suspicion is borne from the fact that the requirement for CypA is both cell type, and species, specific, suggesting that CypA is not required simply to uncoat the core. This notion is further supported by the observation that $\mathrm{CA}$ point mutants close to the CypA binding site such as HIV-1 CA A92E or G94D appears to lead to restriction of HIV-1 in human cells [55,56]. A92E or G94D infectivity is reduced in some human cell lines but not others and strikingly, infectivity is rescued by inhibition of CypA. It is possible that these mutants become sensitive to human restriction factor(s) and that the interaction between the factor and 

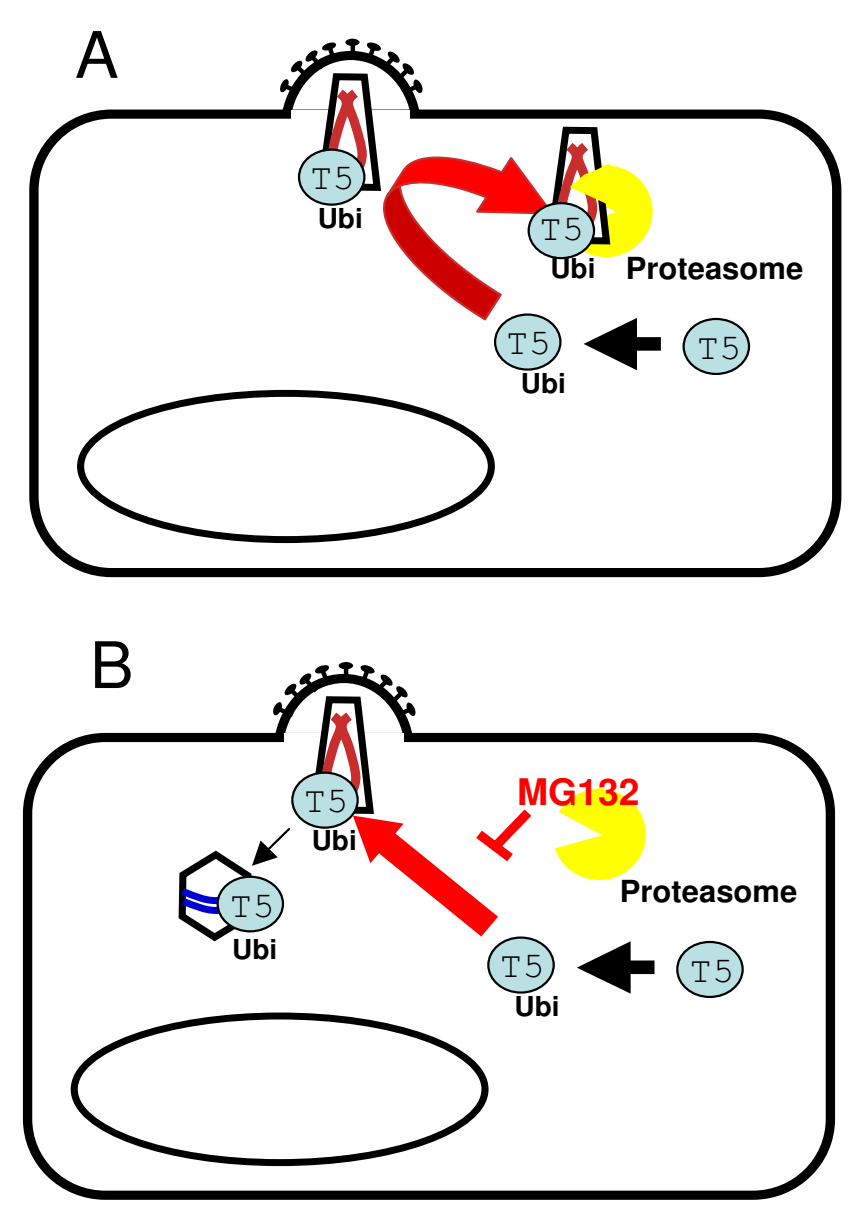

Figure I

A putative mechanism for restriction of retroviruses by TRIM5 $\alpha$. (Panel A) TRIM5 $\alpha$ is autoubiquitinylated in a RING dependent way and rapidly turned over by the proteasome [47]. If it encounters incoming sensitive retroviral cores then they too are recruited to the proteasome and destroyed, before the virus has the opportunity for significant reverse transcription. (Panel B) If the virus/TRIM5 $\alpha$ complex is protected from destruction, by inhibiting the proteasome, then the virus can reverse transcribe [48, 49]. Infectivity is not rescued however, indicating that the virus/ TRIM5 $\alpha$ complex is uninfectious. How TRIM5 renders the virus uninfectious remains unclear.

the virion is sensitive to the activity of CypA on the peptide bond at P90.

How might CypA impact on recognition of CA by TRIM $5 \alpha$ ? One possibility is that in some cases, capsid with CypA attached may make a better target for TRIM5 $\alpha$. This possibilty has been discounted on the basis that HIV-1 mutated to prevent CypA binding (HIV-1 CA G89V) remains restricted by TRIM $5 \alpha$ from Old World monkeys $[59,61]$. Importantly, this mutant is not restricted by TRIM-Cyp, which relies on the CypA domain to recruit it to the HIV-1 capsid [43]. A second possibility is that recruitment of TRIM $5 \alpha$ to capsid is improved by the prolyl isomerisation activity of CypA on HIV-1 capsid. Prolyl isomerisation has been shown to regulate protein-protein interaction in diverse biological systems including the control of cell division by cdc $25 \mathrm{C}$ and signalling by the Itk receptor. The prolyl isomerase Pin 1 catalyses the cis/trans isomerisation of a proline peptide bond in cdc25C. Cdc25C activity is regulated by phosphorylation and since its phosphatase PP2A only recognises the cdc25C trans isomer, Pin1 activity leads to dephosphorylation and cdc25C activation [63]. A similar molecular switch has been described for Itk signalling and CypA. CypA catalyses cis/trans isomerisation of proline 287 in the Itk SH2 domain impacting on interaction with phosphorylated signalling partners and regulating Itk activity $[64,65]$. NMR measurements have shown that HIV-1 CA contains around $86 \%$ trans and $14 \%$ cis at G89-P90 in both the presence and absence of CypA [57]. However, in the presence of CypA, CA is rapidly isomerised between the two states [57]. It is therefore possible that OWM TRIM $5 \alpha$ binds preferentially to CA containing G89-P90 in the cis conformation [59]. In this case, in the presence of TRIM $5 \alpha$, CypA maintains the percentage of cis at $14 \%$ even as TRIM $5 \alpha$ sequesters it from the equilibrium. In this way the trans form is isomerised to cis and becomes bound by TRIM5 $\alpha$. Blocking CypA activity would limit the availability of the cis conformation and therefore TRIM5 $\alpha$ 's ability to see the CA, resulting in rescued infectivity. This model is summarised in Fig 2. CypA also appears to impact on replication of feline immunodeficiency virus in feline and human cells although whether TRIM5 is required for this remains unclear [66].

Surprisingly in the New World species owl monkey a CypA pseudogene has been inserted into the TRIM5 coding region, replacing the viral binding B30.2 domain with CypA, leading to a molecule called TRIMCyp $[43,44]$. This restriction factor strongly restricts HIV-1, SIVagm and FIV by recruitment of the incoming capsid to the RBCC domain facilitated by interaction between the CypA domain and the capsid $[20,66,67]$. Viral infectivity is rescued by inhibition of CypA-CA interactions with CSA indicating the dependence on CypA binding to capsid for robust restriction. We assume that at some point in owl monkey evolution the modification of TRIM5 to TRIMCyp provided a significant selective advantage. We can only speculate on what might have provided the selection pressure but a pathogenic virus that recruited CypA is a possibility. It is worth noting that a TRIMCyp in the human genome would be a useful antiviral as we face the current AIDS pandemic. 


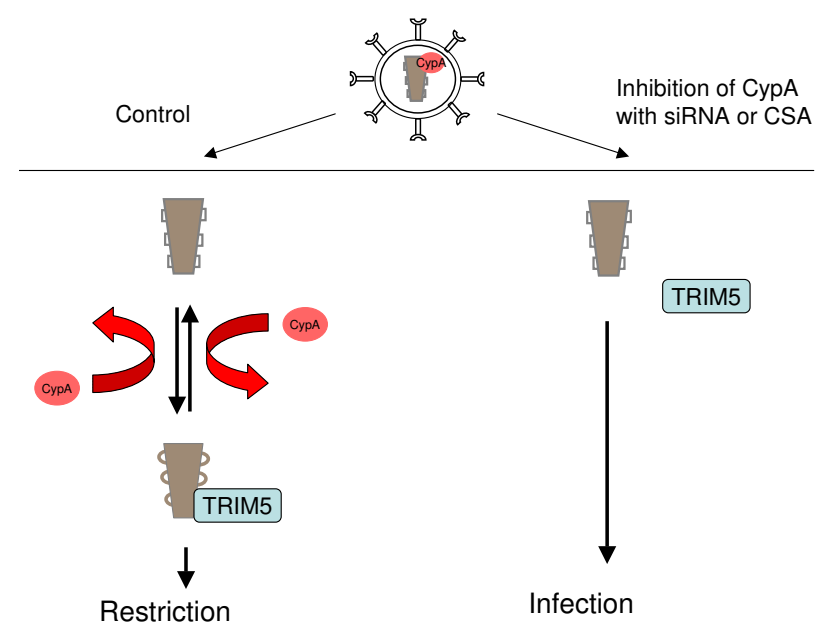

Figure 2

A putative mechanism for activity of CypA on HIV-I infectivity in cells from Old World monkeys. HIV-I recruits CypA to around 10\% of its capsid monomers in newly assembled cores $[52,53]$. When the core enters the cytoplasm of a target cell it recruits more CypA, which efficiently catalyses cis/trans isomerisation of the peptide bond at CA G89-P90 [42, 57]. This activity replenishes the cis conformation $C A$ as it is recruited into the restricted complex with TRIM5 $\alpha$. If CypA activity is reduced in target cells, using CypA specific siRNA or by inhibiting CypA activity with CSA, then the OWM TRIM5 $\alpha$ cannot interact with the CA, which is mostly in the trans conformation, and infectivity is rescued [59-6I]. The isomerisation at CA G89-P90 is represented by squares (trans) changing to circles (cis) on the surface of the capsid.

The role of CypA in sensitivity to TRIM5, its fusion to TRIM5 in owl monkeys and its role as a target for immunosuppression implies that CypA might have a general role in immunity. Viruses are likely to be under considerable pressure to alter their shape and become invisible to antiviral shape recognition systems such as TRIMs. Molecules, such as CypA, that induce shape changing, may have an important role in making escape difficult. For example, HIV-1 appears to be invisible to OWM TRIM5 in the absence of CypA, but in its presence HIV-1 is strongly restricted [59-61]. Conversely, HIV-1 is highly infectious in human cells in the presence of CypA but appears to become restricted in its absence [42]. It seems that HIV-1 is invisible to human TRIM5 whether CypA is active or not but becomes restricted by something else in the absence of CypA activity $[59,62]$. HIV-1 appears to have adapted to tolerate CypA activity and this adaptation has made it dependent on CypA. Why can't HIV-1 simply avoid recruiting CypA? The answer to that is not clear but a clue can be found in alignment of the CypA binding region of lentiviruses (Figure 3). All primate lentiviruses have con- served the proline rich CypA binding loop and many encode glycine proline motifs within it. This suggests that the motifs that recruit CypA are important, conserved and cannot easily be mutated. The loops and glycine proline motifs are also conserved in the equine lentivirus EIAV and the feline FIV [67]. Their purpose however remains unclear and this loop is not conserved in MLV [40] (Figure 4).

\section{Polymorphism and TRIM5 in other species}

The fact that TRIM5 restricts retroviral infection so potently, at least in monkeys, has suggested that polymorphism in human TRIM5 might impact on HIV-1 transmission and/or pathogenesis in vivo. Several studies have addressed this issue and shown at best, only weak association of any particular TRIM $5 \alpha$ allele with disease progression [68-71]. Importantly, human TRIM5 $\alpha$ is not polymorphic in the regions of the B30.2 domain known to impact on viral recognition, and its over expression does not reduce HIV-1 infectivity by more than a few fold $[7,9,10,72]$. Furthermore under in vitro conditions where rhesus TRIM5 efficiently binds the HIV-1 capsid, the human protein binds only poorly [46]. It therefore seems likely that TRIM5 doesn't significantly impact on HIV-1 replication and pathogenesis in humans. Indeed, we imagine that HIV-1's insensitivity to TRIM5 has been an important factor in its success as a pathogen in humans. Conversely the TRIM5 gene in rhesus macaques and sooty mangabeys is relatively polymorphic with a number of polymorphisms occurring in the variable loops that dictate antiviral specificity. Indeed, expression of these alleles in permissive feline cells followed by challenge with retroviral vectors derived from HIV-1, SIVmac MPMV or MLV$\mathrm{N}$ demonstrated that the different alleles have slightly different antiviral specificities [72].

The antiviral activity of TRIMs in mammals other than primates remains less well characterised. A bovine TRIM (BoLv1) with broad anti retroviral activity suggests that TRIM-mediated restriction of retroviruses is widespread amongst mammals [37,38]. BoLv1 is closely related to primate TRIM5 genes suggesting that they are orthologs derived from an ancestral antiviral TRIM. Cattle encode at least 4 genes closely related to TRIM5, in addition to homologs of TRIM34 and TRIM6. The fact that one of these proteins has antiviral activity supports the notion that these genes are derived from an ancestral sequence with antiviral activity. It is likely that antiviral TRIMs will be identified in more mammals soon. Indeed, antiviral TRIMs are probably responsible for the poor infectivity of cells from pigs and bats to MLV-N and those of rabbits to HIV-1 [1,3,5,73]. 
HIV-1 K03455

HIV-1 M62320

HIV-1 U21135

HIV-1 U46016

HIV-1 U88822

HIV-1 AF077336

HIV-1 AF061642

HIV-1 AF005496

HIV-1 AF082394

HIV-1 AJ249239

HIV-1 U54771

HIV-1 L39106

HIV-1 AF193276

HIV-1 AF049337

HIV-1 AJ006022

HIV-1 L20587

HIV-1 L20571

SIVcpz U42720

SIVcpz AF115393

SIVcpz AJ271369

SIVcpz X52154

SIVcpz AF382828

SIVcpz AF447763

SIVcpz AF103818

SIVcol AF301156

SIVdeb AY523865

SIVdeb AY523866

SIVden AJ580407

SIVdrl AY159321

SIVgsn AF468659

SIVgsn AF468658
P-V-HAGPIAPGQMREP

P-V-HAGPIPPGQMREP

P-V-HAGPIAPGQMREP

P-V-HAGPVAPGQMRDP

P-V-QAGPVAPGQMREP

P-V-HAGPAPPGQMREP

P-Q-QAGPFPPGQIREP

P-V-HAGPIPPGQMREP

P-V-HAGPIAPGQVREP

P-V-HAGPIPPGQMREP

P-V-HAGPIPPGQMREP

P-V-HAGPIPPGQMREP

P-A-QAGPF PPGQMREP

P-V-HAGPIPPGOMREP

P-V-PVGPLPPGQLRDP

P-P-PVGPLPPGQIREP

P-P-AMGPLPPGQIREP

P-T-HAGPVQAGQLREP

P-V-HAGPVAPGQLREA

P-V-HAGPIAPGOLREA

P-T-HAGPIAPGQLREP

P-L-HAGPVAPGQMREP

P-A-HAGPQQAGMLREP

P-L- HAGPIAPGOMREP

P-V--QQPOQQGALRQP

P-Q--QQP-PQGQLREP

P-Q--QQP-PQGQLREP

P-ANLQQPLPPAAQRDP

P-Q-V-GPLPQGVLRNP

P-V-PAAPQPVAGLRDP

P-Q-PAAPQPVAGLRDP
HIV-2 AF082339

HIV-2 M30502

HIV-2 M31113

HIV-2 X61240

HIV-2 U27200

HIV-2 AF208027

HIV-2 AY530889

SIVsm AF334679

SIVsm AF077017

SIVstm M83293

SIVmac239 M33262

SIVIst AF188114

SIVIst AF188115

SIVIst AF188116

SIVIst AF075269

SIVmnd AF328295

SIVmnd AF367411

SIVmnd AY159322

SIVmon AY340701

SIVmus AY340700

SIVrcm AF382829

SIVrcm AF349680

SIVgri M66437

SIVsab U04005

SIVtan U58991

SIVver M30931

SIVver L40990

SIVver M29975

SIVver X07805

SIVsun AF131870

SIVsyk L06042

SIVsyk AY523867 $\downarrow$

P-I-P-GPLPAGQLRDP

P-I-P-GPLPAGQLREP

P-S-P-GPMPAGQLRDP

P-S-P-GPMPAGQLREP

P-Q-Q-GPPPAGQXREP

P-N-Q-GPPPAGQLREP

PRG-QQPAQPAGGLREP

P-Q-P-GPIPAGQLRDP

P-Q-PPGPLPAGQLREP

P-Q-PA-P-QQGQLREP

P-Q-QQPAQPGGGLRTP

P-Q-QQPAQPGGGLRTP

P-Q-QQPAQPGGGLRTP

P-Q-QQPAQPGGGLRTP

P-Q-V-GPLPQGVLRNP

P-Q-V-GPMPQGVLRNP

P-P-V-GPMPQGVLRNP

P-QPPQ-PQPNAGLGDP

P-OPOQ-POPNAGLRNP

P-V-P-GPIPAGQLREP

P-N-P-GPLPAGQLREP

R-P-PAGPLPAGQLRDP

P-P-PQQPPAQGVLRDP

P-P-PAGPLPAGQLRDP

P-P-PAGPLPAGQLRDP

P-P-PAGPLPPGQLRDP

P-P-PAGPLPAGQLRDP

P-L-PAGPLPAGQLRDP

P-Q-QQPAQPGGGLRTP

P-Q-PQQPAPQAGLRDP

P-Q-PQQQQPVAGLRDP

Figure 3

Similarity between the sequences of retroviral capsids. Alignment of primate lentiviral capsid protein sequences demonstrates that they have conserved the proline rich Cyclophilin A binding loop on their outer surface. Glycine proline motifs are common (red arrow). Conserved prolines at the extremes of the loop are shown (black arrows). The alignment from which this selection was taken is available from the Los Alamos HIV sequences database [93]. Retroviruses are named according to the species from which they were isolated. Genbank accession numbers are shown. Species abbreviations are as follows: cpz chimpanzee, deb De Brazza's monkey, den Dent's Mona monkey, drl drill, gsn greater spot nosed monkey, sm sooty mangabey, stm stump tailed macaque, mac rhesus macaque, Ist L'Hoest monkey, mnd mandrill, mon Cercopithecus mona, mus Cercopithecus cephus, rcm red capped mangabey, gri African green monkey Grivet, sab African green monkey sabaeus, tan African green monkey tantalus, ver African green monkey vervet, sun sun tailed monkey, syk Sykes monkey.

Are there other TRIMs with antiviral properties?

Protein families arise through the duplication of ancestral gene sequences and therefore members of a family share common ancestry. Human TRIM5 lies on chromosome 11 within a group of closely related TRIMs, comprising TRIMs 5, 6, 34 and 22, which have presumably arisen by gene duplication. These TRIMs, as well as TRIMs 1, 18, 19 and 21 have no, or relatively weak, antiviral activity against a panel of distantly related retroviruses including HIV-1, HIV-2, SIVmac, EIAV and MLV [20]. Whether this is because they have an alternate function or whether they are simply not active against this selection of viruses is difficult to say. It is worth noting however that comparison of the sequences of these TRIMs from primates shows that 


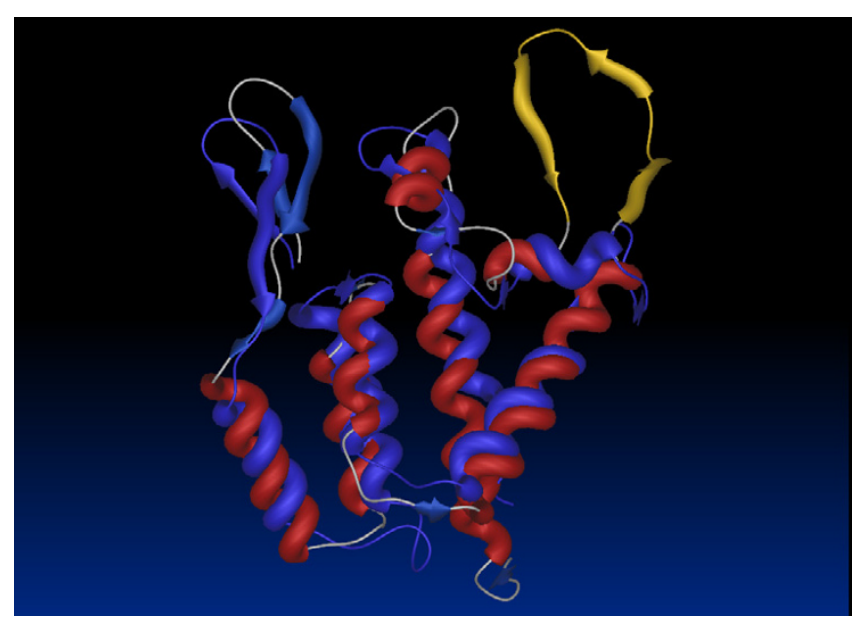

Figure 4

Similarity between the structures of retroviral capsids. Superimposition of the structures of the $\mathrm{N}$ terminal domains of HIV-I (Red) and MLV (blue) capsids demonstrates overall structural conservation although the Cyclophilin A binding loop (yellow) is absent in MLV. The pdb files for HIV-I (IM9C) [94] and MLV (IUK7) [40] were superimposed using pairwise structure comparison [95].

unlike TRIM5, TRIMs 6, 22 and 34 do not have strongly selected B30.2 domains, suggesting that they have not been under the same selection pressures as TRIM5 [74].

There is an increasing body of evidence, gathered over many years suggesting that TRIM19, otherwise known as PML, may have antiviral activity. PML exists in subnuclear structures called PODs, ND10 or PML bodies and are of unclear function. It has long been known that a number of diverse viruses including influenza, SV40 and papilloma virus form replication complexes in close association with PML bodies, reviewed in [75,76]. Infection by other viruses, including herpes viruses and adenoviruses, causes degradation of PML protein and dispersal of the body components. The molecular details of PML degradation by herpes simplex type 1 (HSV-1) have been partially solved. The HSV-1 protein ICPO is responsible for inducing proteasome dependent degradation of $\mathrm{PML}$, and HSV1 deleted for this protein replicates poorly, leaving PML bodies intact [77-80]. Importantly, mutant HSV-1 (ICP0) becomes almost fully infectious if PML expression is reduced using RNA interference, indicating that an important function of ICP0 is to eliminate PML [81]. An antiviral role for PML is also suggested by a real time microscopy study demonstrating that PML is recruited to incoming HSV-1 (ICP0-) replication complexes [82]. Such active recruitment is strongly suggestive of an antiviral response. Furthermore, reduction of PML expression increases permissivity of human cells to human cytomegalovirus infection [83], and over-expression of PML reduces permissivity to vesicular stomatitis virus and influenza A $[84,85]$. These data, along with the observation that PML expression is stimulated by type 1 interferon, strongly support an antiviral role for TRIM19 (PML). Interestingly, PML does not have a B30.2 domain suggesting that it interacts with target viruses in a different way to TRIM $5 \alpha$ interacting with retroviruses.

Further data supporting an antiviral role for TRIM proteins comes from expression studies in which TRIMs are expressed in permissive cells and the modified cells tested for permissivity to infection by retroviral vectors. Such studies have demonstrated weak anti-retroviral activity of TRIM1 from African green monkeys and Owl monkeys against MLV-N [9]. It is also worth noting that a particular TRIM protein can impact on viral infectivity by influencing the activity of another antiviral TRIM protein. For example, expression of TRIM34 can reduce the antiviral activity of TRIM5 presumably via heteromutimerisation mediated via the coiled coil [20]. This observation suggests a complex mechanism of regulation and generation of alternate antiviral specificities through heteromultimerisation. Whether further TRIMs have antiviral activity remains largely untested. The fact that TRIMs 10, 15, 26, $27,31,38,39,40$ are associated with the major histocompatibility complex on chromosome 6 [86] and the observation that the expression of most of these genes is upregulated by influenza infection [87] suggests that they might have a role in immunity.

TRIM20, otherwise known as pyrin, presents as an intriguing antiviral possibility. Polymorphism in the TRIM20 B30.2 domain can cause familial Mediterranean fever, a disease characterised by recurrent attacks of fever and inflammation. Sequencing TRIM20 from a variety of primates revealed that many encode the disease causing mutations as wild type sequence [88]. Furthermore, phylogenetic analysis suggested episodic selection in the B30.2 domain, similar to that seen for TRIM5, suggesting the intriguing possibility that viral infection underlies this disease. Rather strikingly in 2001 these authors suggested that the B30.2 domain of pyrin might interact directly with pathogens and that the mutations are counter evolutionary changes selected to cope with a changing pathogen [88]. Such a model is remarkably close to what we believe to be true for TRIM5, retroviruses and the Red Queen 6 years later.

\section{Concluding Remarks}

Just as we considered that the important aspects of TRIM5 biology had been largely described, the Ikeda lab described tantalising findings that make a complicated subject significantly more complicated [89]. They show that rhesus TRIM5 causes degradation of gag in infected cells. Importantly this activity is independent of the C-ter- 
minal B30.2 domain suggesting that it acts via an alternative specificity determinant, perhaps the coiled coil. It is worth noting that APOBEC $3 \mathrm{G}$ has also been described as being able to restrict infection of both incoming as well as outgoing HIV-1 [90,91]. It may be therefore that such dually active restriction factors are not uncommon.

Whether the study of host factors influencing viral infection will translate into improvements in antiviral therapy in the foreseeable future remains uncertain. However, it is likely to allow the improvement of animal models for HIV/AIDS as we enhance our understanding of the viral and cellular determinants for viral replication and disease [92]. This work is also likely to improve our ability to transduce cells, therapeutically and experimentally, with viral gene delivery vectors, particularly poorly permissive primary cells and stem cells. It certainly promises to remain an active and exciting field in infectious disease research.

\section{Abbreviations}

TRIM, tripartite motif; MLV, murine leukemia virus; MLV$\mathrm{N}, \mathrm{N}$ tropic MLV; MLV-B, B tropic MLV; CypA Cyclophilin A; CSA, cyclosporine A; CA, capsid

\section{Competing interests}

The author(s) declare that they have no competing interests.

\section{Acknowledgements}

Thanks to members of the Towers lab for their contribution to the ideas presented, Laura Ylinen, Zuzana Keckesova, Ben Webb, Shalene Singh, Torsten Schaller, Claire Pardieu and Sam Wilson. Thanks to Daryl Bosco, Brandeis University, Stephan Hue, UCL and Rob Gifford, Stanford University for helpful discussion and Gordon Perkins, Blue Tractor Software and Mike Malim, Kings College London for their input. Our work is funded by the Wellcome Trust, the Medical Research Council UK, the UCL graduate School and the Bogue Fellowship Scheme, UCL.

\section{References}

I. Towers G, Bock M, Martin S, Takeuchi Y, Stoye JP, Danos O: A conserved mechanism of retrovirus restriction in mammals. Proc Natl Acad Sci USA 2000, 97: I 2295-12299.

2. Shibata R, Sakai H, Kawamura M, Tokunaga K, Adachi A: Early replication block of human immunodeficiency virus type $I$ in monkey cells. J Gen Virol 1995, 76:2723-2730.

3. Hofmann W, Schubert D, LaBonte J, Munson L, Gibson S, Scammell J, Ferrigno P, Sodroski J: Species-specific, postentry barriers to primate immunodeficiency virus infection. J Virol 1999, 73:10020-10028.

4. Towers G, Collins M, Takeuchi Y: Abrogation of RefI restriction in human cells. J Virol 2002, 76:2548-2550.

5. Besnier C, Takeuchi $Y$, Towers G: Restriction of lentivirus in monkeys. Proc Natl Acad Sci USA 2002, 99: I 1920 - I 1925.

6. Cowan S, Hatziioannou T, Cunningham T, Muesing MA, Gottlinger HG, Bieniasz PD: Cellular inhibitors with FvI-like activity restrict human and simian immunodeficiency virus tropism. Proc Natl Acad Sci USA 2002, 99:1 1914 -1 1919.

7. Stremlau M, Owens CM, Perron MJ, Kiessling M, Autissier P, Sodroski J: The cytoplasmic body component TRIM5alpha restricts HIV-I infection in Old World monkeys. Nature 2004, 427:848-853.
8. Keckesova Z, Ylinen LM, Towers G]: The human and African green monkey TRIM5alpha genes encode RefI and LvI retroviral restriction factor activities. Proc Natl Acad Sci USA 2004, I0I:10780-10785.

9. Yap MW, Nisole S, Lynch C, Stoye JP: Trim5alpha protein restricts both HIV-I and murine leukemia virus. Proc Natl Acad Sci USA 2004, I01: 10786-1079|.

10. Hatziioannou T, Perez-Caballero D, Yang A, Cowan S, Bieniasz PD: Retrovirus resistance factors Refl and LvI are species-specific variants of TRIM5alpha. Proc Natl Acad Sci USA 2004, I0I: 10774-10779.

II. Perron MJ, Stremlau M, Song B, Ulm W, Mulligan RC, Sodroski J: TRIM5alpha mediates the postentry block to $\mathrm{N}$-tropic murine leukemia viruses in human cells. Proc Natl Acad Sci USA 2004, I01: I I827-I I832.

12. Asaoka K, Ikeda K, Hishinuma T, Horie-Inoue K, Takeda S, Inoue S: A retrovirus restriction factor TRIM5alpha is transcriptionally regulated by interferons. Biochem Biophys Res Commun 2005, 338: 1950-1956.

13. Borden KL, Lally JM, Martin SR, O'Reilly NJ, Etkin LD, Freemont PS: Novel topology of a zinc-binding domain from a protein involved in regulating early Xenopus development. Embo J 1995, 14:5947-5956.

14. Reymond A, Meroni G, Fantozzi A, Merla G, Cairo S, Luzi L, Riganelli $D$, Zanaria E, Messali S, Cainarca S, et al.: The tripartite motif family identifies cell compartments. Embo / 200I, 20:2I40-2I5I.

15. Xu L, Yang L, Moitra PK, Hashimoto K, Rallabhandi P, Kaul S, Meroni G, Jensen JP, Weissman AM, D'Arpa P: BTBDI and BTBD2 colocalize to cytoplasmic bodies with the RBCC/tripartite motif protein, TRIM5delta. Exp Cell Res 2003, 288:84-93.

16. Massiah MA, Matts JA, Short KM, Simmons BN, Singireddy S, Yi Z, Cox TC: Solution Structure of the MIDI B-box2 CHC(DI C) C(2)H(2) Zinc-binding Domain: Insights into an Evolutionarily Conserved RING Fold. J Mol Biol 2007, 369: I- 10.

17. Massiah MA, Simmons BN, Short KM, Cox TC: Solution structure of the RBCC/TRIM B-boxl domain of human MIDI: B-box with a RING. J Mol Biol 2006, 358:532-545.

18. Perez-Caballero D, Hatziioannou T, Yang A, Cowan S, Bieniasz PD: Human Tripartite Motif 5\{alpha\} Domains Responsible for Retrovirus Restriction Activity and Specificity. J Virol 2005, 79:8969-8978.

19. Mische CC, Javanbakht H, Song B, Diaz-Griffero F, Stremlau M, Strack $\mathrm{B}, \mathrm{Si} \mathrm{Z}$, Sodroski J: Retroviral restriction factor TRIM5alpha is a trimer. J Virol 2005, 79: I4446-14450.

20. Zhang F, Hatziioannou T, Perez-Caballero D, Derse D, Bieniasz PD: Antiretroviral potential of human tripartite motif-5 and related proteins. Virology 2006.

21. Yap MW, Nisole S, Stoye JP: A Single Amino Acid Change in the SPRY Domain of Human Trim5alpha Leads to HIV-I Restriction. Curr Biol 2005, I 5:73-78.

22. Stremlau M, Perron MJ, Welikala S, Sodroski J: Species-specific variation in the B30.2(SPRY) domain of TRIM5alpha determines the potency of human immunodeficiency virus restriction. J Virol 2005, 79:3।39-3।45.

23. Passerini LD, Keckesova Z, Towers GJ: Retroviral Restriction Factors FvI and TRIM5\{alpha\} Act Independently and Can Compete for Incoming Virus before Reverse Transcription. J Virol 2006, 80:2100-2105.

24. Rhodes DA, de Bono B, Trowsdale J: Relationship between SPRY and B30.2 protein domains. Evolution of a component of immune defence? Immunology 2005, I 16:4|I-4I7.

25. Perron MJ, Stremlau M, Sodroski J: Two surface-exposed elements of the B30.2/SPRY domain as potency determinants of $\mathrm{N}$-tropic murine leukemia virus restriction by human TRIM5alpha. J Virol 2006, 80:563I-5636.

26. Nakayama EE, Miyoshi H, Nagai Y, Shioda T: A Specific Region of 37 Amino Acid Residues in the SPRY (B30.2) Domain of African Green Monkey TRIM5\{alpha\} Determines Species-Specific Restriction of Simian Immunodeficiency Virus SIVmac Infection. J Virol 2005, 79:8870-8877.

27. Sebastian S, Luban J: TRIM5alpha selectively binds a restrictionsensitive retroviral capsid. Retrovirology 2005, 2:40.

28. Stremlau M, Perron M, Lee M, Li Y, Song B, Javanbakht H, DiazGriffero F, Anderson DJ, Sundquist WI, Sodroski J: Specific recognition and accelerated uncoating of retroviral capsids by the 
TRIM5alpha restriction factor. Proc Natl Acad Sci USA 2006, 103:55|4-55|9.

29. Grutter C, Briand C, Capitani G, Mittl PR, Papin S, Tschopp J, Grutter MG: Structure of the PRYSPRY-domain: implications for autoinflammatory diseases. FEBS letters 2006, 580:99-106.

30. Woo JS, Imm JH, Min CK, Kim KJ, Cha SS, Oh BH: Structural and functional insights into the B302/SPRY domain. Embo J 2006.

31. James LC, Keeble AH, Khan Z, Rhodes DA, Trowsdale J: Structural basis for PRYSPRY-mediated tripartite motif (TRIM) protein function. Proc Natl Acad Sci USA 2007, 104:6200-6205.

32. Ohkura S, Yap MW, Sheldon T, Stoye JP: All three variable regions of the TRIM5alpha B30.2 domain can contribute to the specificity of retrovirus restriction. J Virol 2006, 80:8554-8565

33. Sawyer SL, Wu LI, Emerman M, Malik HS: Positive selection of primate TRIM5alpha identifies a critical species-specific retroviral restriction domain. Proc Natl Acad Sci USA 2005 , 102:2832-2837.

34. Lively CM, Dybdahl MF: Parasite adaptation to locally common host genotypes. Nature 2000, 405:679-68I.

35. Katzourakis A, Tristem M, Pybus OG, Gifford RJ: From the Cover: Discovery and analysis of the first endogenous lentivirus. Proc Natl Acad Sci USA 2007, 104:626I-6265.

36. Hatziioannou T, Cowan S, Goff SP, Bieniasz PD, Towers GJ: Restriction of multiple divergent retroviruses by LvI and RefI. Embo J 2003, 22: I- I0.

37. Ylinen LM, Keckesova Z, Webb BL, Gifford RJ, Smith TP, Towers GJ: Isolation of an Active LvI Gene from Cattle Indicates that Tripartite Motif Protein-Mediated Innate Immunity to Retroviral Infection Is Widespread among Mammals. J Virol 2006, 80:7332-7338

38. Si Z, Vandegraaff N, O'Huigin C, Song B, Yuan W, Xu C, Perron M, Li $X$, Marasco WA, Engelman A, et al.: Evolution of a cytoplasmic tripartite motif (TRIM) protein in cows that restricts retroviral infection. Proc Natl Acad Sci USA 2006, 103:7454-7459.

39. Li S, Hill CP, Sundquist WI, Finch JT: Image reconstructions of helical assemblies of the HIV-I CA protein. Nature 2000, 407:409-4I3.

40. Mortuza GB, Haire LF, Stevens A, Smerdon SJ, Stoye JP, Taylor IA: High-resolution structure of a retroviral capsid hexameric amino-terminal domain. Nature 2004, 43 I:48I-485.

41. Ylinen L, Keckesova Z, Wilson SJ, Ranasinghe S, Towers G]: Differential restriction of HIV-2 and SIVmac by TRIM5alpha alleles. J Virol 2005, 79: I I 580-I I 587.

42. Towers G], Hatziioannou T, Cowan S, Goff SP, Luban J, Bieniasz PD: Cyclophilin A modulates the sensitivity of HIV-I to host restriction factors. Nat Med 2003, 9: I I 38- I I 43 .

43. Sayah DM, Sokolskaja E, Berthoux L, Luban J: Cyclophilin A retrotransposition into TRIM5 explains owl monkey resistance to HIV-I. Nature 2004, 430:569-573.

44. Nisole S, Lynch C, Stoye JP, Yap MW: A Trim5-cyclophilin A fusion protein found in owl monkey kidney cells can restrict HIV-I. Proc Natl Acad Sci USA 2004, I0I: I3324-I3328.

45. Javanbakht H, Yuan W, Yeung DF, Song B, Diaz-Griffero F, Li Y, Li X, Stremlau M, Sodroski J: Characterization of TRIM5alpha trimerization and its contribution to human immunodeficiency virus capsid binding. Virology 2006, 353:234-246.

46. Stremlau M, Perron M, Lee M, Li Y, Song B, Javanbakht H, DiazGriffero F, Anderson DJ, Sundquist WI, Sodroski J: From the Cover: Specific recognition and accelerated uncoating of retroviral capsids by the TRIM5\{alpha\} restriction factor. Proc Natl Acad Sci USA 2006, 103:55।4-5519.

47. Diaz-Griffero F, Li X, Javanbakht H, Song B, Welikala S, Stremlau M, Sodroski J: Rapid turnover and polyubiquitylation of the retroviral restriction factor TRIM5. Virology 2006, 349(2):300-I5.

48. Wu X, Anderson JL, Campbell EM, Joseph AM, Hope TJ: Proteasome inhibitors uncouple rhesus TRIM5alpha restriction of HIV-I reverse transcription and infection. Proc Natl Acad Sci USA 2006, 103:7465-7470.

49. Anderson JL, Campbell EM, Wu X, Vandegraaff N, Engelman A, Hope TJ: Proteasome inhibition reveals that a functional preintegration complex intermediate can be generated during restriction by diverse TRIM5 proteins. I Virol 2006, 80:9754-9760.

50. Perron MJ, Stremlau M, Lee M, Javanbakht H, Song B, Sodroski J: The Human TRIM5\{alpha\} Restriction Factor Mediates Acceler- ated Uncoating of the N-Tropic Murine Leukemia Virus Capsid. J Virol 2007, 8I:2I 38-2।48.

5I. Chatterji U, Bobardt MD, Gaskill P, Sheeter D, Fox H, Gallay PA: Trim5alpha accelerates degradation of cytosolic capsid associated with productive HIV-I entry. J Biol Chem 2006.

52. Thali M, Bukovsky A, Kondo E, Rosenwirth B, Walsh CT, Sodroski J, Gottlinger HG: Functional association of cyclophilin A with HIV-I virions. Nature 1994, 372:363-365

53. Franke EK, Yuan HE, Luban J: Specific incorporation of cyclophilin A into HIV-I virions. Nature 1994, 372:359-362.

54. Kootstra NA, Munk C, Tonnu N, Landau NR, Verma IM: Abrogation of postentry restriction of HIV-I-based lentiviral vector transduction in simian cells. Proc Natl Acad Sci USA 2003, 100:1298-1303

55. Hatziioannou T, Perez-Caballero D, Cowan S, Bieniasz PD: Cyclophilin interactions with incoming human immunodeficiency virus type I capsids with opposing effects on infectivity in human cells. I Virol 2005, 79: |76-|83.

56. Sokolskaja E, Sayah DM, Luban J: Target cell cyclophilin A modulates human immunodeficiency virus type I infectivity. J Virol 2004, 78: $12800-12808$.

57. Bosco DA, Eisenmesser EZ, Pochapsky S, Sundquist WI, Kern D: Catalysis of cis/trans isomerization in native HIV-I capsid by human cyclophilin A. Proc Natl Acad Sci USA 2002, 99:5247-5252.

58. Bosco DA, Kern D: Catalysis and binding of cyclophilin A with different HIV-I capsid constructs. Biochemistry 2004, 43:61I0-6119.

59. Keckesova Z, Ylinen L, Towers G]: Cyclophilin A renders HIV-I sensitive to old world monkey but not human TRIM5a antiviral activity. J Virol 2006, 80:4683-4690.

60. Berthoux L, Sebastian S, Sokolskaja E, Luban J: Cyclophilin A is required for TRIM5\{alpha\}-mediated resistance to HIV-I in Old World monkey cells. Proc Natl Acad Sci USA 2005, I02: | 4849- | 4853

61. Stremlau M, Song B, Javanbakht H, Perron M, Sodroski J: Cyclophilin A: An auxiliary but not necessary cofactor for TRIM5alpha restriction of HIV-I. Virology 2006, 35 I: I I2-I20.

62. Sokolskaja E, Berthoux L, Luban J: Cyclophilin A and TRIM5alpha independently regulate human immunodeficiency virus type I infectivity in human cells. I Virol 2006, 80:2855-2862.

63. Zhou XZ, Kops O, Werner A, Lu PJ, Shen M, Stoller G, Kullertz G, Stark M, Fischer G, Lu KP: Pin I-dependent prolyl isomerization regulates dephosphorylation of $\mathrm{Cdc} 25 \mathrm{C}$ and tau proteins. Mol Cell 2000, 6:873-883.

64. Brazin KN, Mallis RJ, Fulton DB, Andreotti AH: Regulation of the tyrosine kinase Itk by the peptidyl-prolyl isomerase cyclophilin A. Proc Natl Acad Sci USA 2002, 99:1899-1904.

65. Colgan J, Asmal M, Neagu M, Yu B, Schneidkraut J, Lee Y, Sokolskaja $E$, Andreotti $A$, Luban J: Cyclophilin A regulates TCR signal strength in CD4+ $T$ cells via a proline-directed conformational switch in Itk. Immunity 2004, 2 I: | 89-20I.

66. Lin TY, Emerman M: Cyclophilin A interacts with diverse lentiviral capsids. Retrovirology 2006, 3:70.

67. Diaz-Griffero F, Vandegraaff N, Li Y, McGee-Estrada K, Stremlau M, Welikala S, Si Z, Engelman A, Sodroski J: Requirements for capsidbinding and an effector function in TRIMCyp-mediated restriction of HIV-I. Virology 2006, 35 I (2):404-I9.

68. Speelmon EC, Livingston-Rosanoff D, Li SS, Vu Q, Bui J, Geraghty DE, Zhao LP, McElrath MJ: Genetic association of the antiviral restriction factor TRIM5alpha with human immunodeficiency virus type I infection. J Virol 2006, 80:2463-247I.

69. Goldschmidt V, Bleiber G, May M, Martinez R, Ortiz M, Telenti A Role of common human TRIM5alpha variants in HIV-I disease progression. Retrovirology 2006, 3:54.

70. Javanbakht H, An P, Gold B, Petersen DC, O'Huigin C, Nelson GW O'Brien SJ, Kirk GD, Detels R, Buchbinder S, et al.: Effects of human TRIM5alpha polymorphisms on antiretroviral function and susceptibility to human immunodeficiency virus infection. Virology 2006, 354:15-27.

7I. Sawyer SL, Wu LI, Akey JM, Emerman M, Malik HS: High-Frequency Persistence of an Impaired Allele of the Retroviral Defense Gene TRIM5alpha in Humans. Curr Biol 2006, 16:95-100.

72. Newman RM, Hall L, Connole M, Chen GL, Sato S, Yuste E, Diehl W, Hunter E, Kaur A, Miller GM, Johnson WE: Balancing selection and the evolution of functional polymorphism in Old World 
monkey TRIM5alpha. Proc Natl Acad Sci USA 2006 , 103:19134-19139.

73. Besnier C, Ylinen L, Strange B, Lister A, Takeuchi Y, Goff SP, Towers $\mathrm{G}$ : Characterization of murine leukemia virus restriction in mammals. J Virol 2003, 77:13403-13406.

74. Li X, Gold B, O'Huigin C, Diaz-Griffero F, Song B, Si Z, Li Y, Yuan W, Stremlau M, Mische C, et al:: Unique features of TRIM5alpha among closely related human TRIM family members. Virology 2007, 360(2):419-33.

75. Everett RD: DNA viruses and viral proteins that interact with PML nuclear bodies. Oncogene 200I, 20:7266-7273.

76. Everett RD, Chelbi-Alix MK: PML and PML nuclear bodies: Implications in antiviral defence. Biochimie 2007.

77. Chelbi-Alix MK, de The $\mathrm{H}$ : Herpes virus induced proteasomedependent degradation of the nuclear bodies-associated PML and Sp I00 proteins. Oncogene 1999, I8:935-94I.

78. Everett RD, Freemont P, Saitoh H, Dasso M, Orr A, Kathoria M, Parkinson J: The disruption of NDI 0 during herpes simplex virus infection correlates with the VmwII0- and proteasomedependent loss of several PML isoforms. J Virol 1998 72:658|-659|

79. Everett RD, Boutell C, Orr A: Phenotype of a herpes simplex virus type I mutant that fails to express immediate-early regulatory protein ICP0. J Virol 2004, 78: I763-I774.

80. Hagglund R, Roizman B: Role of ICPO in the strategy of conquest of the host cell by herpes simplex virus I. I Virol 2004 78:2169-2178

81. Everett RD, Rechter S, Papior P, Tavalai N, Stamminger T, Orr A: PML contributes to a cellular mechanism of repression of herpes simplex virus type $I$ infection that is inactivated by ICPO. J Virol 2006, 80:7995-8005.

82. Everett RD, Murray J: ND I 0 components relocate to sites associated with herpes simplex virus type I nucleoprotein complexes during virus infection. J Virol 2005, 79:5078-5089.

83. Tavalai N, Papior P, Rechter S, Leis M, Stamminger T: Evidence for a role of the cellular NDIO protein PML in mediating intrinsic immunity against human cytomegalovirus infections. Virol 2006, 80:8006-8018.

84. Chelbi-Alix MK, Quignon F, Pelicano L, Koken MH, de The H: Resistance to virus infection conferred by the interferon-induced promyelocytic leukemia protein. J Virol 1998, 72:1043-105I.

85. Iki S, Yokota S, Okabayashi T, Yokosawa N, Nagata K, Fujii N Serum-dependent expression of promyelocytic leukemia protein suppresses propagation of influenza virus. Virology 2005, 343: 106-115.

86. Nisole S, Stoye JP, Saib A: TRIM family proteins: retroviral restriction and antiviral defence. Nat Rev Microbiol 2005 3:799-808.

87. Geiss GK, Salvatore M, Tumpey TM, Carter VS, Wang X, Basler CF, Taubenberger JK, Bumgarner RE, Palese P, Katze MG, Garcia-Sastre A: Cellular transcriptional profiling in influenza $A$ virusinfected lung epithelial cells: the role of the nonstructural NSI protein in the evasion of the host innate defense and its potential contribution to pandemic influenza. Proc Natl Acad Sci USA 2002, 99:10736-10741.

88. Schaner P, Richards N, Wadhwa A, Aksentijevich I, Kastner D, Tucker $P$, Gumucio D: Episodic evolution of pyrin in primates: human mutations recapitulate ancestral amino acid states. Nat Genet 200I, 27:3|8-32I.

89. Sakuma R, Noser JA, Ohmine S, Ikeda Y: Rhesus monkey TRIM5alpha restricts HIV-I production through rapid degradation of viral Gag polyproteins. Nat Med 2007.

90. Chiu YL, Soros VB, Kreisberg JF, Stopak K, Yonemoto W, Greene WC: Cellular APOBEC3G restricts HIV-I infection in resting CD4+ T cells. Nature 2005, 435:108-1/4.

91. Pion M, Granelli-Piperno A, Mangeat B, Stalder R, Correa R, Steinman $\mathrm{RM}$, Piguet $\mathrm{V}$ : APOBEC3G/3F mediates intrinsic resistance of monocyte-derived dendritic cells to HIV-I infection. J Exp Med 2006, 203:2887-2893.

92. Hatziioannou T, Princiotta M, Piatak M Jr, Yuan F, Zhang F, Lifson JD, Bieniasz PD: Generation of simian-tropic HIV-I by restriction factor evasion. Science 2006, 3। 4:95.

93. HIV sequences database [http://www.hiv.lanl.gov]

94. Yoo S, Myszka DG, Yeh C, McMurray M, Hill CP, Sundquist WI: Molecular recognition in the HIV-I capsid/cyclophilin A complex. J Mol Biol 1997, 269:780-795.
95. CATH Protein Structure Classification
Publish with Bio Med Central and every scientist can read your work free of charge

"BioMed Central will be the most significant development for disseminating the results of biomedical research in our lifetime. "

Sir Paul Nurse, Cancer Research UK

Your research papers will be:

- available free of charge to the entire biomedical community

- peer reviewed and published immediately upon acceptance

- cited in PubMed and archived on PubMed Central

- yours - you keep the copyright 